\title{
Efficient in situ detection of mRNAs using the Chlorella virus DNA ligase for padlock probe ligation
}

\author{
NILS SCHNEIDER ${ }^{1,2}$ and MATTHIAS MEIER ${ }^{1,2}$ \\ ${ }^{1}$ Microfluidic and Biological Engineering, Department of Microsystems Engineering_IMTEK, University of Freiburg, 79110 Freiburg, Germany \\ ${ }^{2}$ Centre for Biological Signalling Studies-BIOSS, University of Freiburg, 79104 Freiburg, Germany
}

\begin{abstract}
Padlock probes are single-stranded DNA molecules that are circularized upon hybridization to their target sequence by a DNA ligase. In the following, the circulated padlock probes are amplified and detected with fluorescently labeled probes complementary to the amplification product. The hallmark of padlock probe assays is a high detection specificity gained by the ligation reaction. Concomitantly, the ligation reaction is the largest drawback for a quantitative in situ detection of mRNAs due to the low affinities of common DNA or RNA ligases to RNA-DNA duplex strands. Therefore, current protocols require that mRNAs be reverse transcribed to DNA before detection with padlock probes. Recently, it was found that the DNA ligase from Paramecium bursaria Chlorella virus 1 (PBCV-1) is able to efficiently ligate RNA-splinted DNA. Hence, we designed a padlock probe assay for direct in situ detection of mRNAs using the PBCV-1 DNA ligase. Experimental single-cell data were used to optimize and characterize the efficiency of mRNA detection with padlock probes. Our results demonstrate that the PBCV-1 DNA ligase overcomes the efficiency limitation of current protocols for direct in situ mRNA detection, making the PBCV-1 DNA ligase an attractive tool to simplify in situ ligation sequencing applications.
\end{abstract}

Keywords: DNA padlock probes; in situ ligation; mRNA quantification

\section{INTRODUCTION}

Padlock probes are single-stranded DNA molecules for in situ detection of specific DNA or RNA sequences. For this purpose, a padlock probe contains two annealing sites at the strand ends, which are complementary to the target sequence. Upon hybridization of the padlock probe to its target, the $5^{\prime}$ and $3^{\prime}$ ends become adjacent and available for an intramolecular ligation reaction. In the following, the circular padlock probe acts as a template for isothermal rolling circle amplification (RCA). RCA products are detected with short fluorescently labeled oligonucleotides complementary to the linker region spacing the annealing sites of the padlock probe (Banér et al. 1998). The two main advantages of this nucleotide detection technology reside in the (i) high sequence-specific enzymatic ligation reaction and (ii) strong DNA signal amplification. Unlike fluorescence in situ hybridization (FISH) methods, padlock probes are less prone to false-positive signals resulting from nonspecific binding or adhesion of probes within fixed tissues and cells. Therefore, the padlock probe assay and its modifications have applications in the detection of single-nucleotide polymorphisms (SNPs) in the genome (Hardenbol et al. 2003), mitochondrial DNA (Larsson et al. 2004), and/or microRNAs.

Corresponding author: matthias.meier@imtek.de

Article is online at http://www.rnajournal.org/cgi/doi/10.1261/rna. 057836.116
Although the padlock assay is attractive for many analytical applications, the low activity of commercially available DNA ligases for DNA/RNA hybrids hampers the use of this technology for direct in situ detection of mRNAs. For example, the bacteriophage T4 DNA ligase is able to initiate the ligation reaction of RNA-splinted DNA by firstly AMPylating the phosphorylated DNA donor strand. Slow reaction kinetics and weak binding affinities of the T4 DNA ligase for the RNA/DNA hybrid causes dissociation of the T4 ligase from the RNA/DNA strand before the DNA nick is sealed. Furthermore, the AMPylated donor strand is blocked and excluded from further ligation reactions (Nilsson et al. 2001; Bullard and Bowater 2006).

To bypass this problem, mRNAs are reverse transcribed (RT) into cDNA fragments, and then detected with padlock probes targeting the cDNA rather than the corresponding mRNA (Larsson et al. 2010). This RT-based approach has been successfully applied to generate a gene expression profile of breast cancer tissue (Ke et al. 2013); however, the disadvantage of this method is the requirement of expensive locked-nucleic-acid (LNA) modified primers to transcribe

(C) 2017 Schneider and Meier This article is distributed exclusively by the RNA Society for the first 12 months after the full-issue publication date (see http://rnajournal.cshlp.org/site/misc/terms.xhtml). After 12 months, it is available under a Creative Commons License (Attribution-NonCommercial 4.0 International), as described at http://creativecommons.org/licenses/bync/4.0/. 
high quantities of mRNA. Furthermore, after the RT, the remaining mRNA needs to be degraded with an additional enzymatic step in order to make the cDNA accessible to the padlock probe. In consequence of the complex padlock probe assay structure, other single molecule or self-assembling branched FISH probe assays have gained momentum as alternatives for the in situ and absolute quantification of mRNAs (Lubeck and Cai 2012; Battich et al. 2013).

Recently, it was found that the Chlorella virus DNA ligase (PBCV-1 DNA ligase) can ligate RNA-splinted DNA with unexpectedly high efficiency (Lohman et al. 2014). In a RNA ligation assay with Jurkat total RNA cell extracts, the PBCV-1 DNA ligase displayed approximately 100 -fold higher efficiency for an RNA-splinted DNA ligation reaction than the most commonly used T4 ligase.

Herein, we demonstrate that, upon parameter optimization, the PBCV-1 DNA ligase can be used for direct in situ detection of mRNA transcripts without the need of RT. For this purpose, we designed a padlock probe assay and detected $\beta$-actin mRNA within single HeLA cells. In a quantitative comparison between the PBCV-1 DNA ligase and the T4 DNA ligase in cell populations of over 1000 cells, we demonstrate the applicability of the approach for rapid and specific detection of mRNAs.

\section{RESULTS}

\section{In situ padlock probe ligation with the PBCV-1 DNA ligase}

First, we aimed to determine whether padlock probes hybridized to mRNAs could be ligated in situ by the PBCV-1 DNA ligase. For this purpose, a padlock probe against the $5^{\prime}$-end of human $\beta$-actin mRNA (ATCB) was designed (see Fig. 1A). The padlock probe, hereafter named PP-1, was added to fixed HeLa cells at a concentration of $1 \mu \mathrm{M}$ and ligated with 200 $\mathrm{nM}$ PBCV-1 DNA ligase at $37^{\circ} \mathrm{C}$ for $30 \mathrm{~min}$. Additionally, in a negative control experiment, a modified padlock probe (PP-1n) was added to fixed cells under the same conditions. $\mathrm{PP}-1 \mathrm{n}$ is identical to PP- 1 with the exception of one base pair mismatch $(\mathrm{A} \rightarrow \mathrm{T})$ at the $3^{\prime}$ end to the ACTB target. The $3^{\prime}$ end of the padlock probe is defined as the acceptor site, and the phosphorylated $5^{\prime}$ end as the donor site. In general, ligases exhibit highest sensitivity for base pair mismatches at the $3^{\prime}$ acceptor site (Sriskanda and Shuman 1998; Tong et al. 1999, 2000).

In situ ligated padlock probes were amplified with a phi29 polymerase in an RCA step. To provide a double-stranded starting region for the phi29, a primer was added. This differs from the padlock probe assays using the RT strategy, where the $3^{\prime}$ to $5^{\prime}$ exonuclease activity of phi29 is able to cut back the single-stranded CDNA, until it acts as a primer for the RCA reaction. This is not possible for padlock probes hybridizing directly to mRNAs, because secondary structure elements of mRNAs effectively inhibit the phi29 exonuclease
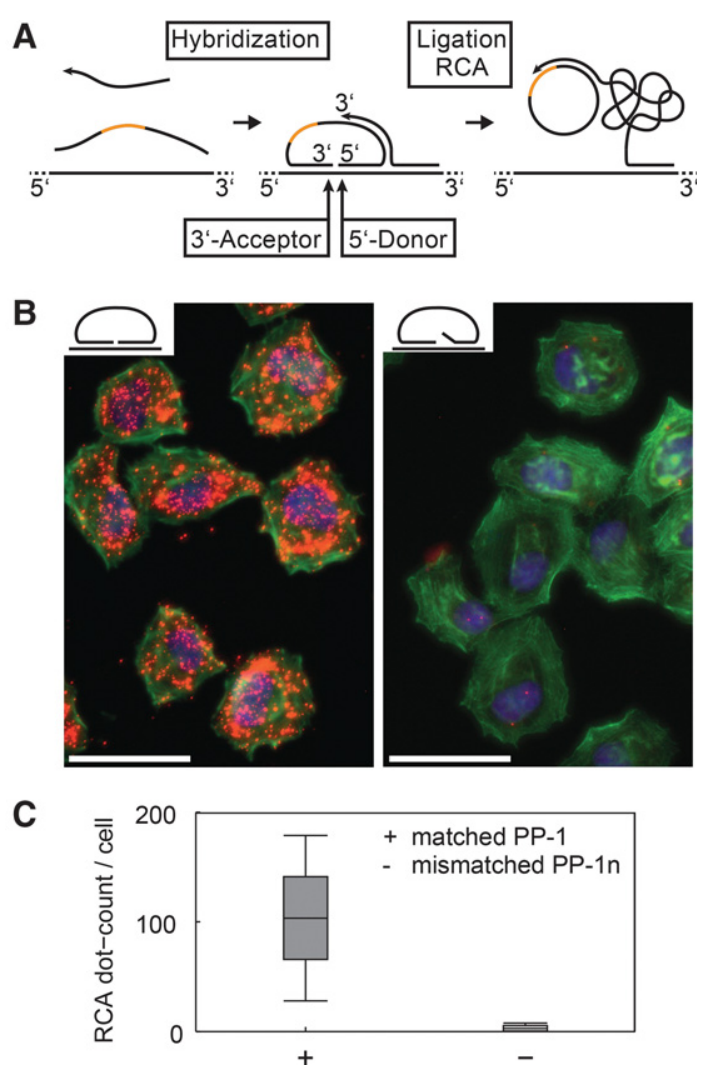

FIGURE 1. In situ detection of mRNAs using the PBCV-1 DNA ligase. (A) Reaction schematic: (i) The padlock probe and RCA primer hybridize to the fixed mRNA target. (ii) RNA splinted padlock probe is ligated by the PBCV-1 DNA ligase. (iii) The padlock probe is amplified within a primer-initiated RCA reaction, and detected with fluorescence probe complementary to a probe binding sequence (orange). (B) Representative image of HeLa cells with PP-1 (left) and PP-1n (right) after the padlock probe assay. Red, green, and blue are the counterstains for the RCA product, cytoskeleton, and nucleus, respectively. The scale bar represents $50 \mu \mathrm{M}$. (C) Box plot of the RCA dot count per cell obtained for the PP-1 and PP-1n padlock probe in 1000 HeLa cells. The whisker shows the cell-to-cell variation of the median RCA dot count per cell, whereas the outside bars show $2 \sigma$.

activity. Only upon addition of RNase III, which degrades double-stranded mRNA, the additional primer can be omitted, to perform a direct mRNA detection assay with padlock probes (Merkiene et al. 2010). The additional enzymatic reaction step was avoided by using a primer. The primer was 44 nucleotides (nt) long, of which $20 \mathrm{nt}$ (located at the $3^{\prime}$-end) were complementary to the intermediate padlock probe sequence. After a short 4-nt linker, the $20 \mathrm{nt}$ located at the $5^{\prime}$ end were complementary to the adjacent hybridization region of the padlock probe on the mRNA sequence to anchor the RCA product to the mRNA.

Figure $1 \mathrm{~B}$ shows representative images of cells subjected to the padlock probe assay using the PBCV-1 DNA ligase for the target matched PP-1 (left) and mismatched PP-1n (right) probe. The mean RCA dot count number per single cell for PP-1 and PP-n was 94 and 3, respectively. Both values were calculated with a $95 \%$ confidence level and a sample size of 

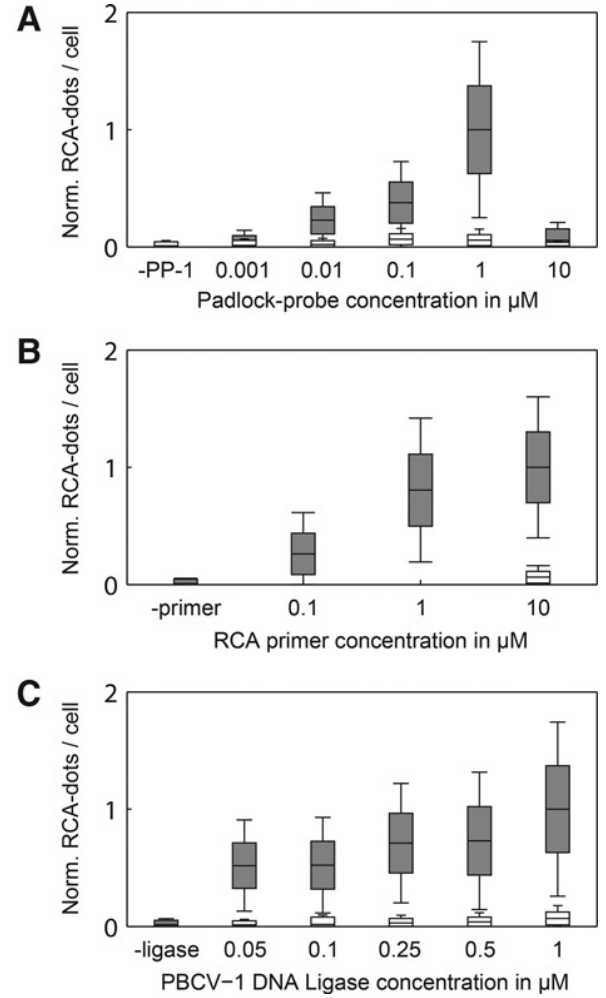

FIGURE 2. Parameter optimization of the padlock probe assay for detection of in situ mRNAs using the PBCV-1 DNA ligase. Variations in the RCA dot count per cell upon changing the padlock probe $(A)$, RCA primer $(B)$, PBCV-1 DNA ligase concentration $(C)$. All other reaction parameters were left constant. Gray and white box plots denote PP-1 and PP-1n, respectively.

1000 cells from three replicates (Fig. 1C). This result demonstrated that PBVC-1 is able to specifically ligate in situ DNA padlock probes hybridized to mRNAs.

\section{Optimization of the in situ ligation conditions for the PBCV-1 DNA ligase}

In the second step, we optimized the padlock probe ligationassay conditions for the PBCV-1 DNA ligase. We investigated the effects of the padlock probe, primer, and ligase concentrations on the in situ RCA dot count per cell. Unless the conditions were varied, $200 \mathrm{nM}$ of PBCV-1 DNA ligase, $1 \mu \mathrm{M}$ of padlock probe PP-1 or PP-1n, and the RCA-primer were used. For a comparison of the different reaction conditions, we normalized the RCA dot count per cell to the condition with the maximum RCA dot count.

Figure 2A shows the normalized RCA dot count per cell upon increasing the padlock probe concentration. A logarithmic increase of the PP-1 concentration ( $1 \mathrm{pM}$ to $1 \mu \mathrm{M})$ led to a 20-fold increase in the normalized RCA dot counts per cell. At the same time, the increase of the padlock probe concentration resulted in a fourfold improvement in the PP-1/PP-1n ratio of RCA dot counts per cell. At a concentration of $10 \mu \mathrm{M}$, the PP-1 signal significantly dropped to a level comparable to that of $1 \mathrm{nM}$ of a padlock probe.

Figure $2 \mathrm{~B}$ shows that a gradient increase of primer concentration $(100 \mathrm{nM}, 1 \mu \mathrm{M}$, and $10 \mu \mathrm{M})$ resulted in a fourfold increase of RCA dot counts per cell. Without addition of the primer to the padlock probe assay, no RCA signal could be detected. Concomitantly, the low background level for PPin did not increase by increasing the primer concentration up to $10 \mu \mathrm{M}$. We also tested a shorter RCA primer that hybridized only to the padlock probe and not the mRNA target and found that RCA dot counts per cell and distribution were comparable (data not shown).

Figure 2C shows the RCA dot count per cell upon increasing the PBCV-1 ligase concentration. An increase from 50 $\mathrm{nM}$ to $500 \mathrm{nM}$ led to only a 1.4 -fold increase in dot count. The signal-to-noise ratio, which is defined as the PP-1/PP1n ratio of RCA dot count per cell, was constant within the PBCV-1 DNA ligase concentration series. At a ligase concentration of $1 \mu \mathrm{M}$, we noticed that clusters of RCA products started to form outside of cells on the cell culture substrate, indicating nonspecific ligation events. In a control reaction without PBCV-1 DNA ligase, no RCA signal was detected in the cells.

Furthermore, the reaction kinetics of the PBCV-1 DNA ligase were characterized within the in situ padlock probe assay. Variations in the ligation time for PP- 1 from 0.5 to $24 \mathrm{~h}$ did not change the RCA dot count per cell for the PP-1 padlock probe.

\section{Padlock probe efficiency depends on the targeted mRNA sequence position}

It has been previously shown that targeting the $5^{\prime}$-end of the ACTB mRNA with a padlock probe using the RT strategy with LNA modified primers, results in a RCA dot count per cell of approximately 120-310 within four different cell lines (Larsson et al. 2010). Therefore, our direct DNA/RNA hybridization approach with PP-1 showed a slightly lower efficiency. A possible explanation could be that the hybridization sequence of PP-1 on the ACTB mRNA is blocked by local RNA structure elements or bound proteins. Another possibility is that the PBCV-1 DNA ligase exhibits a bias for particular base pairs at the ligation junction of the padlock probe. In fact, the PBCV-1 DNA ligase exhibits different ligation rates for RNA-splinted DNA where A/T bases at the donor site are preferred over G/C nucleotides (Lohman et al. 2014).

To investigate the influence of the nucleotides at the ligation junction of PPs on the mRNA detection efficiency, we designed three additional PPs (PP-2, 3, and 4) with different donor/acceptor pairs (Fig. 3A). The hybridization site of the three PPs was shifted by only 1 nucleotide in order to exclude a localization effect. Figure $3 \mathrm{~B}$ shows representative images of cells subjected to the direct padlock probe assay using PP-2 to 4. The obtained RCA dot counts per cell for all PPs are given 
A

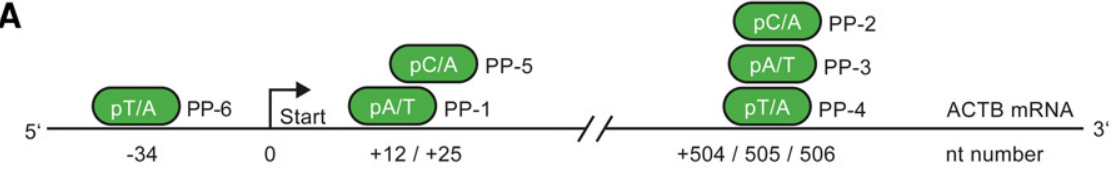

B
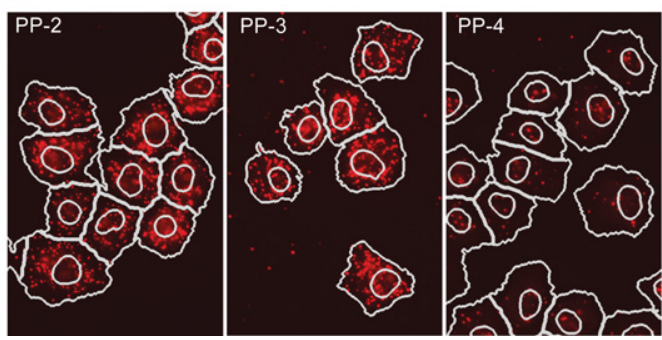

$\mathrm{C}$

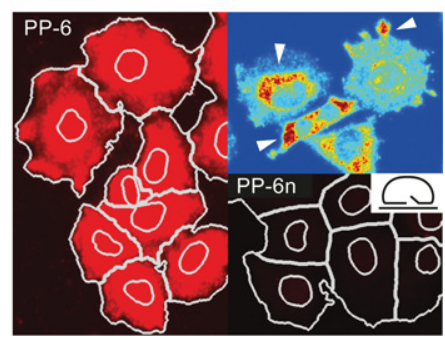

FIGURE 3. Efficiency of the padlock probe assay using the PBCV-1 DNA ligase is dependent on the target position and nucleotide sequence. (A) Schematic of the ATCB mRNA sequence with indicated hybridization region of the six padlock probes (PP). The donor/acceptor nucleotide pair of each PP is given in the green filled circles. $(B, C)$ Representative images of cells subjected to the padlock probe assay targeting the ATCB mRNA with the PP-2, 3, 4, and 6. Outlines of the cells and nucleus are obtained by image segmentation. equally efficiently ligated as the A/T pair of PP-1 and 3. At a PP-6 concentration of $1 \mu \mathrm{M}$, the RCA dot count number was too high for quantification, due to overlapping fluorescence signals in the cell images (Fig. 3C). The corresponding negative control padlock probe, $\mathrm{PP}-6 n$, showed no RCA product, which confirmed the specificity of the RCA signal. Notably, the distribution of RCA dots detected with PP-6 appeared to be cellstate dependent. Migrating and spreading cells after division displayed obvious agglomerations of RCA signals in budding lamellipodia (colored Fig. 3C). To be able to compare PP-6 quantitatively to the other PPs we repeated the assay with a 10 -fold lower PP-6 concentration. The result is added to Table 1 . in Table 1. The assays revealed that PP-2 with a C/A donor/ acceptor pair exhibited a 50\% lower RCA dot count per cell, than PP-3 and 4 with a T/A or A/T donor/acceptor pair at the ligation junction, respectively. To confirm this result we designed PP-5, which targeted the ATCB mRNA close to the hybridization site of PP-1 with an equal hybridization energy. In contrast to PP-1, PP-5 contained a C/A donor/acceptor pair. In line with the results for PP- 2 to 4 the RCA dot count per cell for PP-5 was clearly reduced compared to PP-1 (see Table 1).

In the next step, we investigated the influence of the PP hybridization site on the RCA dot count with the direct padlock probe assay. For this it was possible to compare the assay results of PP-1 and PP-3, which primed 500 nt downstream from PP-1. A threefold lower RCA dot count per cell for PP-3 than PP-1 was observed. The difference cannot be explained by a nucleotide bias of the PBCV-1 DNA ligase since both PPs contained an A/T donor/acceptor pair. For further exploration of the localization effect of the PP hybridization site we designed PP- 6 , which targeted the $5^{\prime}$ UTR region of the ATCB mRNA and thus upstream of PP-1. PP- 6 contained a T/A donor/acceptor pair, for which we showed that it is

\section{Comparison between PBCV-1 and T4 DNA ligase for in situ ligation of RNA-splinted DNA}

Finally, we compared the performance of the PBCV-1 DNA ligase to the T4 DNA ligase for in situ ligation of the six padlock probes and their negative controls. For this purpose, the concentrations of the PBCV-1 and T4 DNA ligases were adjusted to a protein concentration of $200 \mathrm{nM}$.

The box plot in Figure 4 shows the representative results for PP-1, PP-5, and PP-6. To perform a direct comparison of the RCA dot counts for each padlock probe with the two ligases, the results were normalized to the result obtained with the PBCV-1 DNA ligase. For PP-1, both ligases produced a comparable RCA dot count per cell, whereas the negative padlock probe $(\mathrm{PP}-1 n)$ resulted in a 2.5 -fold increase in the background signal using the T4 DNA ligase.

For PP-5, a sixfold increase in the RCA dot count per cell was obtained when ligated with the PBCV-1 DNA ligase, compared to the ligation with T4 ligase. Whereas for PBCV-1 DNA ligase, the signal-to-noise ratio was about six, for T4 DNA Ligase it was $\sim 0.4$. This result is explained by the design of the PP-5n padlock probe sequence. PP-5n exhibits a G/U

TABLE 1. Summary of the padlock probe results

\begin{tabular}{|c|c|c|c|c|c|c|c|c|c|c|}
\hline Padlock probe (PP) & 1 & $1 n$ & 2 & 3 & $3 n$ & 4 & 5 & $5 n$ & $6^{\mathrm{a}}$ & $6 n$ \\
\hline Donor/acceptor pair & $\mathrm{pA} / \mathrm{T}$ & $\mathrm{pA} / \mathrm{A}$ & $\mathrm{pC} / \mathrm{A}$ & $\mathrm{pA} / \mathrm{T}$ & $\mathrm{pA} / \mathrm{A}$ & $\mathrm{pT} / \mathrm{A}$ & $\mathrm{pC} / \mathrm{A}$ & $\mathrm{pC} / \mathrm{G}$ & $\mathrm{pT} / \mathrm{A}$ & $\mathrm{pT} / \mathrm{T}$ \\
\hline Mean RCA dots/cell & 94 & 3 & 15 & 33 & 3 & 29 & 29 & 6 & 219 & 4 \\
\hline Cell-to-cell variation of RCA dots/cell & 31 & 1 & 8 & 18 & 2 & 15 & 12 & 5 & 92 & 4 \\
\hline Standard deviation of RCA dots/cell between experiments & 20 & 1 & 4 & 12 & 1 & 8 & 12 & 3 & 38 & 2 \\
\hline
\end{tabular}

The cell-to-cell variability was determined as standard deviation of the RCA dot count between at least 1000 cells within one experiment. The standard deviation for the experimental repeat was determined from three independent experiments.

${ }^{a}$ The mean dot count per cell was obtained with a 100-nM PP-6 concentration. 


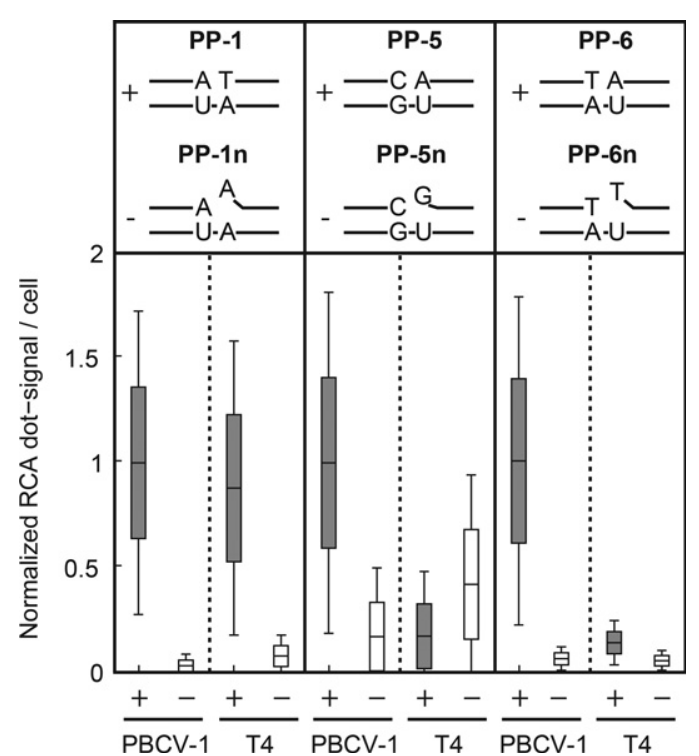

FIGURE 4. Comparison of the PBCV-1 DNA ligase with the T4 ligase within an in situ mRNA detection with padlock probes. The box plot shows the RCA dot count per cell for the three representative padlock probes for the ATCB mRNA and their negative controls. RCA dot counts are normalized to the result of the PBCV-1 DNA ligase.

mismatch at the acceptor side. Guanine is able to form a wobble base pair with uracil, which is difficult to be distinguished from matched G/C by the T4 DNA ligase (Jang et al. 2015).

Consistent with the above findings, we observed a high RCA dot count per cell for PP-6 using the PBCV-1 DNA ligase at a padlock probe concentration of $1 \mu \mathrm{M}$. In order to obtain a quantitative RCA dot count per cell for PP-6, the padlock probe concentration was lowered by a factor of 10 , whereas all other parameters remained constant. A 7.5-fold higher efficiency for the PBCV-1 DNA ligase was found compared to the T4 ligase. Notably, extrapolation of the RCA dot count per cell result for the PBCV-1 to the 10 times higher PP-2 probe concentration would argue for an absolute count number of $2000-3000$ ATCB mRNA in a single HeLa cell, which matches previous single molecule FISH results (Latham et al. 1994) in fibroblast cells.

All experiments were carried out in triplicates with at least 2600 cells per condition and verified in independent experiments. In addition, we also tested the hypothesis that the T4 DNA ligase performs better when the ATP concentration is lowered to $10 \mu \mathrm{M}$. For the T4 DNA ligase, a decrease in the ATP concentration led to an $\sim 90 \%$ drop in the RCA dot count per cell, when compared to the results at high ATP concentration. For the PBCV-1 DNA ligase, the decrease in the ATP concentration did not change the RCA dot count per cell (data not shown).

\section{DISCUSSION}

DNA ligation probes have repeatedly been used for in vitro detection of mRNAs, including micro RNAs (Nilsson et al.
2000; Jonstrup et al. 2006; Li et al. 2009). One major obstacle for direct in situ mRNA detection using padlock probes is their low detection efficiency compared to alternative approaches with subcellular resolution such as single molecule FISH, branched FISH DNA probes, or hybridization chain reaction probes (Crosetto et al. 2015). The main reason for this low detection efficiency is the lack of ligases for ligation events of RNA-splinted DNA (Nilsson et al. 2001). It has been determined that $\sim 1 \%$ of total cellular mRNA transcripts are detectable with DNA ligation probes (Lagunavicius et al. 2009; Larsson et al. 2010). Despite the efficiency problem, DNA ligation probes are still desirable for mRNA detection because of their high specificity accompanied by a low false-positive signal compared to other hybridization probes.

Herein, we demonstrate that the Chlorella virus DNA-ligase can overcome the limitation of ligase efficiency for direct in situ mRNA detection using padlock probes, by retaining the high specificity of the assay. The characterization of the assay showed that mRNA in situ detection is dependent on both the padlock probe and primer concentration. This is expected because the hybridization probability of the padlock probe with the mRNA and the primer with the ligated padlock probe are increased with the number of available DNA strands. The reason why an increase in the concentration of the padlock probe (over $1 \mu \mathrm{M}$ ) led to a drop in the number of detected mRNAs is unclear; however, it is likely caused by either padlock probe aggregation or depletion of the ligase through nonspecifically hybridized padlock probes. The RCA read out signal of the padlock probe assay showed no significant changes upon increasing the PBCV-1 DNA ligase concentration form $50 \mathrm{nM}$ to $1 \mu \mathrm{M}$. Furthermore, the RCA signal was stable at ligation times longer than $30 \mathrm{~min}$. These in situ results are in agreement with the previous findings for RNA-splinted DNA ligation reactions with the PBCV-1 DNA ligase in a solution assay. Notably, the presented protocol does not include any particularly stringent wash steps.

It has been previously demonstrated that the PBCV-1 DNA ligase exhibits a lower ligation and higher AMPylation rate for RNA-splinted DNA with a C or G at the donor site. The comparison of PP-2, 3, and 4 with the same hybridization region on the ATCB mRNA but a different donor/acceptor pair at the ligation junction confirmed the PBCV-1 nucleotide bias under the in situ conditions. Only the PP containing a $\mathrm{C}$ at the donor site (PP-2) showed reduced detection efficiency compared to the PPs containing either an $\mathrm{A}$ (PP-3) or T (PP-4) at the donor site.

To clarify whether the in situ detection of mRNA with the PBCV-1 DNA ligase is dependent on the position on the mRNA sequence, three padlock probes against different regions of the ACTB mRNA were compared. The designed $\mathrm{PP}-1,2$, and 6 targeted exon 1 , exon 2 , and the $5^{\prime}$ UTR region of the ACTB mRNA, respectively. PP-6 resulted in the highest RCA dot count per cell, whereas the RCA signal for PP-1 and 2 strongly dropped. Direct detection of mRNA with a ligation probe requires that the targeted sequence is accessible and 
TABLE 2. Padlock probe DNA sequences

\begin{tabular}{|c|c|}
\hline Padlock probe & DNA sequence \\
\hline PP-1 & $\begin{array}{l}\text { [Phos]ATCATCATCCATGGTGAGCACCAGCGATCTGCGAGACCGTATGCCACATCGCTTCCTAGTTCTGATCCGACGA } \\
\text { CGAGCGCGGCGAT }\end{array}$ \\
\hline PP-1_Primer & CATCCATGGTGAGCTGGCGGGTCTGAATACGGTCTCGCAGATCG \\
\hline PP-2 & $\begin{array}{l}\text { [Phos]CCCCTCGTAGATGGGCACAGTGAATGCGAGTCCGTCTAACCATCAATGTCGTTAGTGTAGGTGCATGGGGG } \\
\text { AGGGCATA }\end{array}$ \\
\hline PP-3 & $\begin{array}{l}\text { [Phos]ACCCCTCGTAGATGGGCCAGTGAATGCGAGTCCGTCTAACCATCAATGTCGTTAGTGTAGGTGGCA } \\
\text { TGGGGGAGGGCAT }\end{array}$ \\
\hline PP-4 & $\begin{array}{l}\text { [Phos] TACCCCTCGTAGATGGGCAGTGAATGCGAGTCCGTCTAACCATCAATGTCGTTAGTGTAGGTTGGCA } \\
\text { TGGGGGAGGGCA }\end{array}$ \\
\hline PP-2/3/4_Primer & GCCAGCCAGGTCCAGACGCACGTACCACTAACGACATTGATGGTT \\
\hline PP-5 & $\begin{array}{l}\text { [Phos]CGAGCGCGGCGATATCATCTAAAGCGATCTCCGAGACCGTATGCCACATCGCTTCCTAGTTCTGTACCC } \\
\text { GGAGCCGTTGTCGACGA }\end{array}$ \\
\hline PP-5_Primer & 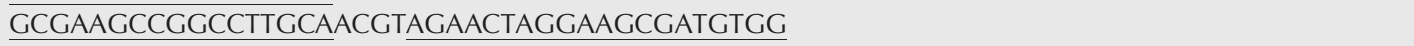 \\
\hline PP-6 & [Phos]ICGGCAAAGGCGAGGTTCAGTCTACGAAGCGATCTGCGAGACCGTATTCTTCTTGTGGACGGGCGGCGGA \\
\hline PP-6_Primer & CATCCATGGTGAGCTGGCGGGTCTGAATACGGTCTCGCAGATCG \\
\hline
\end{tabular}

Underlined, italic, and bold sequences denote the hybridization site on the ATCB mRNA, the probe detection site, and the donor/acceptor ligation pair, respectively. DNA sequences of the negative control padlock probes exhibited the same DNA sequences as the positive padlock probes with the difference that the acceptor base was exchanged as follows: PP1n $(T \rightarrow A)$, PP3n $(T \rightarrow A)$, PP5n $(A \rightarrow C)$, and PP6n $(A \rightarrow T)$.

not blocked by folded secondary RNA structure elements or mRNA binding proteins. In general, fluorescent mRNA probes are preferentially designed against the start codon region of mRNA, because there is a lower probability of finding secondary structures in this region (Rhee et al. 2008). On the other side, global ribosome profiling has shown that ribosomes frequently stall at the start codon and the fifth codon (Han et al. 2014). Therefore, the start codon region of an mRNA with high transcription rates will be blocked for the hybridization of padlock probes. Padlock probes designed against the $5^{\prime}$ and $3^{\prime}$ ends of reversed transcribed mRNAs did not show a dependency on sequence position within the transcript (Larsson et al. 2010). In conclusion, for obtaining high RCA count numbers it is necessary to test several mRNA sequence positions, an approach not unusual for optimization of hybridization probes (Bao et al. 2009). In cases where the detection efficiency of mRNAs using the direct padlock probe assay is high, it is possible to gain detailed subcellular location profiles of the mRNA. This is demonstrated for the ACTB mRNA with PP-6, where the RCA signal accumulated in lamellipodia of migrating cells, an observation that has been reported before (Shestakova et al. 2001; Lionnet et al. 2011; Katz et al. 2012).

In the last section of this study, we compared the efficiency of the PBCV-1 DNA ligase with the T4 ligase within an in situ mRNA detection reaction. For all designed PPs, the PBCV-1 DNA ligase showed at least threefold higher RCA dot counts per cell for the ATCB mRNA than the T4 ligase. Only in the case of PP-1 were both ligases comparable; however, the T4 ligase showed a higher false positive signal for the negative control padlock probe compared to the PBCV-1 DNA ligase.

In conclusion, efficient in situ detection of mRNAs without the need of an RT step significantly reduces the experimental time down to $3.5 \mathrm{~h}$ (from cell fixation to imaging).
Furthermore, the presented approach does not require any specific oligonucleotides, for example, LNA modifications, thus simplifying the assay. The characteristics of the PBCV1 DNA ligase within in situ mRNA detection applications suggests that the ligase can be applied for quantitative mRNA detection and in future experiments to in situ ligation sequencing approaches to massively multiplex mRNA detection.

\section{MATERIALS AND METHODS}

\section{Cell culture}

HeLa Kyoto cells were cultured according to standard protocols in high-glucose Dulbecco's modified Eagle's medium (DMEM) supplemented with $10 \%$ fetal bovine serum (FBS) and 1\% penicillin/ streptomycin. Padlock assays were carried out in 384-well microplates with glass bottoms (Corning). Prior to cell seeding, wells were incubated with $25 \mu \mathrm{g} / \mathrm{mL}$ human fibronectin (Sigma) diluted in PBS for $30 \mathrm{~min}$. Cells were then trypsinized and resuspended in DMEM at a concentration of $0.15-0.2 \times 10^{6}$ cells $/ \mathrm{mL}$, and $10 \mu \mathrm{L}$ of cell suspension was applied to each well. After a 10 min pre-incubation, the total volume of DMEM was increased to $60 \mu \mathrm{L}$ per well and cells grown overnight at $37^{\circ} \mathrm{C}$ and $5 \% \mathrm{CO}_{2}$.

\section{Padlock probe assay}

Cells were fixed in $4 \%$ formaldehyde (Thermo Scientific) for $15 \mathrm{~min}$ and permeabilized in $0.25 \%$ Triton X-100 (Sigma) for $15 \mathrm{~min}$. After Triton X-100 removal, cells were immediately incubated in 10-15 $\mu \mathrm{L}$ ligation solution. Unless otherwise indicated, the ligation solution was composed of $200 \mathrm{nM}$ PBCV-1 DNA ligase or $200 \mathrm{nM}$ T4 DNA ligase (NEB), $1 \times$ T4 ligase buffer $(50 \mathrm{mM}$ Tris- $\mathrm{HCl}$, $10 \mathrm{mM} \mathrm{MgCl}, 1 \mathrm{mM}$ ATP, $10 \mathrm{mM}$ DTT), $1 \mu \mathrm{M}$ padlock probe (Table 2) (5'-phosphorylated), and $1 \mu \mathrm{M}$ RCA primer diluted in RNase-free water. Low-ATP buffer contained $50 \mathrm{mM}$ Tris- $\mathrm{HCl}$, 
$10 \mathrm{mM} \mathrm{MgCl}_{2}, 10 \mu \mathrm{M}$ ATP, and $10 \mathrm{mM}$ DTT. Ligations were run in duplicates or triplicates for every condition for $30 \mathrm{~min}$, followed by three washes with PBS $+0.05 \%$ Tween (PBS-T). Cells were then incubated at $37^{\circ} \mathrm{C}$ for $90 \mathrm{~min}$ in $7 \mu \mathrm{L}$ amplification mixture containing $1 \mathrm{U} / \mu \mathrm{L}$ phi29 polymerase (Fermentas), $1 \times$ phi29 buffer, $0.25 \mathrm{mM}$ dNTPs (NEB), $0.2 \mu \mathrm{g} / \mu \mathrm{L}$ BSA (NEB), and $5 \%$ glycerol. After two washes in PBS-T, RCA products, cytoskeleton, and nuclei were stained with $10 \mathrm{nM}$ detection probe ([Cy3]CAGTGAATGCGA GTCCGTCT[Cy3] for PP-1/5/6 or [Cy3]CAGTGAATGCGAGT CCGTCT[Cy3] for PP-2/3/4), $2 \times$ saline-sodium citrate (SSC), $0.05 \%$ Tween, $2.5 \mu \mathrm{g} / \mathrm{mL}$ sonicated herring sperm DNA, $25 \mathrm{nM}$ Phalloidin-Atto 488 (Sigma), and $1 \mu \mathrm{g} / \mathrm{mL} 4^{\prime}$,6-diamidino-2-phenylindole dihydrochloride (Sigma) for $30 \mathrm{~min}$ at $37^{\circ} \mathrm{C}$, and finally washed three times in PBS-T to remove residual probe molecules.

\section{Image acquisition and analysis}

For every well, at least 750 cells were imaged with an Axio Observer inverted fluorescence microscope and an AxioCam MRm camera at $20 \times$ magnification. Image sets included nuclei (21HE filter), cytoskeleton (38HE), and RCA signal (43HE); integration times were adjusted manually and kept constant for the entire experiments. Single cell dot counts were acquired by image analysis (Blazek et al. 2013) with the MATLAB Image Processing Toolbox (Mathworks).

\section{ACKNOWLEDGMENTS}

This study was supported by the Excellence Initiative of the German Federal and State Governments (EXC-294) and the German Research Foundation (Emmy-Noether Grant ME3823/1-1).

Received June 6, 2016; accepted November 18, 2016.

\section{REFERENCES}

Banér J, Nilsson M, Mendel-Hartvig M, Landegren U. 1998. Signal amplification of padlock probes by rolling circle replication. Nucleic Acids Res 26: 5073-5078.

Bao G, Rhee WJ, Tsourkas A. 2009. Fluorescent probes for live-cell RNA detection. Annu Rev Biomed Eng 11: 25-47.

Battich N, Stoeger T, Pelkmans L. 2013. Image-based transcriptomics in thousands of single human cells at single-molecule resolution. Nat Methods 10: 1127-1133.

Blazek M, Betz C, Hall MN, Reth M, Zengerle R, Meier M. 2013. Proximity ligation assay for high-content profiling of cell signaling pathways on a microfluidic chip. Mol Cell Proteomics 12: 3898-3907.

Bullard DR, Bowater RP. 2006. Direct comparison of nick-joining activity of the nucleic acid ligases from bacteriophage T4. Biochem J 398: 135-144.

Crosetto N, Bienko M, van Oudenaarden A. 2015. Spatially resolved transcriptomics and beyond. Nat Rev Genet 16: 57-66.

Han Y, Gao X, Liu B, Wan J, Zhang X, Qian SB. 2014. Ribosome profiling reveals sequence-independent post-initiation pausing as a signature of translation. Cell Res 24: 842-851.

Hardenbol P, Banér J, Jain M, Nilsson M, Namsaraev EA, KarlinNeumann GA, Fakhrai-Rad H, Ronaghi M, Willis TD,
Landegren U, et al. 2003. Multiplexed genotyping with sequencetagged molecular inversion probes. Nat Biotechnol 21: 673-678.

Jang EK, Yang M, Pack SP. 2015. Highly-efficient T4 DNA ligase-based SNP analysis using a ligation fragment containing a modified nucleobase at the end. Chem Commun 51: 13090-13093.

Jonstrup SP, Koch J, Kjems J. 2006. A microRNA detection system based on padlock probes and rolling circle amplification. RNA 12: 1747-1752.

Katz ZB, Wells AL, Park HY, Wu B, Shenoy SM, Singer RH. 2012. $\beta$-Actin mRNA compartmentalization enhances focal adhesion stability and directs cell migration. Genes Dev 26: 1885-1890.

Ke R, Mignardi M, Pacureanu A, Svedlund J, Botling J, Wahlby C, Nilsson M. 2013. In situ sequencing for RNA analysis in preserved tissue and cells. Nat Methods 10: 857-860.

Lagunavicius A, Merkiene E, Kiveryte Z, Savaneviciute A, ZimbaiteRuskuliene V, Radzvilavicius T, Janulaitis A. 2009. Novel application of phi29 DNA polymerase: RNA detection and analysis in vitro and in situ by target RNA-primed RCA. RNA 15: 765-771.

Larsson C, Koch J, Nygren A, Janssen G, Raap AK, Landegren U, Nilsson M. 2004. In situ genotyping individual DNA molecules by target-primed rolling-circle amplification of padlock probes. Nat Methods 1: 227-232.

Larsson C, Grundberg I, Söderberg O, Nilsson M. 2010. In situ detection and genotyping of individual mRNA molecules. Nat Methods 7: 395-397.

Latham VM, Kislauskis EH, Singer RH, Ross AF. 1994. $\beta$-actin mRNA localization is regulated by signal transduction mechanisms. J Cell Biol 126: 1211-1219.

Li J, Yao B, Huang H, Wang Z, Sun C, Fan Y, Chang Q, Li S, Wang X, Xi J. 2009. Real-time polymerase chain reaction microRNA detection based on enzymatic stem-loop probes ligation. Anal Chem 81: 5446-5451.

Lionnet T, Czaplinski K, Darzacq X, Shav-Tal Y, Wells AL, Chao JA, Park HY, de Turris V, Lopez-Jones M, Singer RH. 2011. A transgenic mouse for in vivo detection of endogenous labeled mRNA. Nat Methods 8: 165-170.

Lohman GJS, Zhang Y, Zhelkovsky AM, Cantor EJ, Evans TC. 2014. Efficient DNA ligation in DNA-RNA hybrid helices by Chlorella virus DNA ligase. Nucleic Acids Res 42: 1831-1844.

Lubeck E, Cai L. 2012. Single-cell systems biology by super-resolution imaging and combinatorial labeling. Nat Methods 9: 743-748.

Merkiene E, Gaidamaviciute E, Riauba L, Janulaitis A, Lagunavicius A. 2010. Direct detection of RNA in vitro and in situ by target-primed RCA: the impact of E. coli RNase III on the detection efficiency of RNA sequences distanced far from the $3^{\prime}$-end. RNA 16: 1508-1515.

Nilsson M, Barbany G, Antson DO, Gertow K, Landegren U. 2000. Enhanced detection and distinction of RNA by enzymatic probe ligation. Nat Biotechnol 18: 791-793.

Nilsson M, Antson DO, Barbany G, Landegren U. 2001. RNA-templated DNA ligation for transcript analysis. Nucleic Acids Res 29: 578-581.

Rhee WJ, Santangelo PJ, Jo H, Bao G. 2008. Target accessibility and signal specificity in live-cell detection of BMP-4 mRNA using molecular beacons. Nucleic Acids Res 36: e30.

Shestakova EA, Singer RH, Condeelis J. 2001. The physiological significance of $\beta$-actin mRNA localization in determining cell polarity and directional motility. Proc Natl Acad Sci 98: 7045-7050.

Sriskanda V, Shuman S. 1998. Specificity and fidelity of strand joining by Chlorella virus DNA ligase. Nucleic Acids Res 26: 3536-3541.

Tong J, Cao WG, Barany F. 1999. Biochemical properties of a high fidelity DNA ligase from Thermus species AK16D. Nucleic Acids Res 27: 788-794.

Tong J, Barany F, Cao WG. 2000. Ligation reaction specificities of an $\mathrm{NAD}^{+}$-dependent DNA ligase from the hyperthermophile Aquifex aeolicus. Nucleic Acids Res 28: 1447-1454. 

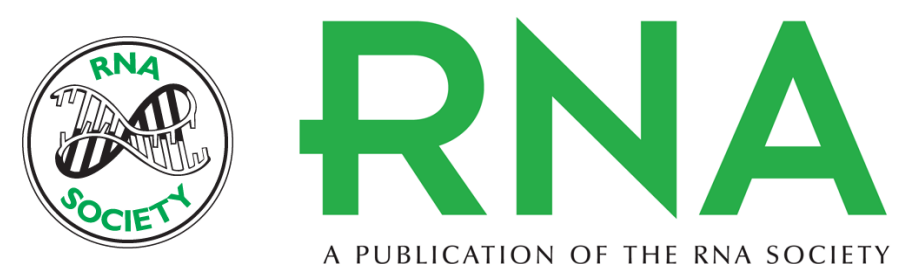

A PUBLICATION OF THE RNA SOCIETY

\title{
Efficient in situ detection of mRNAs using the Chlorella virus DNA ligase for padlock probe ligation
}

\author{
Nils Schneider and Matthias Meier
}

RNA 2017 23: 250-256 originally published online November 22, 2016

Access the most recent version at doi:10.1261/rna.057836.116

\section{References This article cites 28 articles, 8 of which can be accessed free at: http://rnajournal.cshlp.org/content/23/2/250.full.html\#ref-list-1 \\ Creative This article is distributed exclusively by the RNA Society for the first 12 months after the Commons full-issue publication date (see http://rnajournal.cshlp.org/site/misc/terms.xhtml). After 12 License months, it is available under a Creative Commons License (Attribution-NonCommercial 4.0 International), as described at http://creativecommons.org/licenses/by-nc/4.0/.}

Email Alerting Receive free email alerts when new articles cite this article - sign up in the box at the Service top right corner of the article or click here. 